\title{
ATRIBUIÇÕES DA ENFERMAGEM COM OS CUIDADORES DOS PRATICANTES EQUOTERÁPICOS DE CERES - GOIÁS.
}

\section{NURSING ASSIGNMENTS WITH THE CAREGIVERS OF THE EQUERAPIC \\ PRACTITIONERS OF CERES - GOIÁS}

\section{Roberta Rodrigues Guimarães}

Discente do curso de Enfermagem, Faculdade Evangélica de Ceres - GO

beta_guimas@hotmail.com

Gabriela Escorce

Discente do curso de Enfermagem, Faculdade Evangélica de Ceres - GO gescorce@gmail.com

Renata Sousa Nunes

Docente do curso de Enfermagem, Faculdade Evangélica de Ceres - GO renatafisio8@hotmail.com

Endereço para correspondência: Av. Brasil, S/N, Qd.13 Setor Morada Verde, Ceres - GO, Brasil. CEP: 763000-000 Fone: (62) 3323-1040 e-mail: renatafisio8@ hotmail.com

\section{RESUMO}

INTRODUÇÃO: A equoterapia tem um vasto campo de atuação, destinando-se à pacientes portadores de deficiências sensoriais, mentais e motoras, além de se destinar também àpessoas que não se adaptam á sociedade. OBJETIVOS: Ressaltar a importância das atribuições da enfermagem na orientação aos cuidadores dos praticantes da equoterapia no Centro Equoterápico de Ceres - GO; evidenciar a importância do acompanhante ao paciente; orientar os cuidadores sobre as características da patologia, bem como o cuidado continuado fora do Centro de Equoterapia e elaborar planos de cuidados e educação em saúde. METODOLOGIA: Foi adotado o método de pesquisa qualitativa, de caráter exploratório, onde consideramos o mais apropriado para o tipo de análise que pretendemos realizar. As principais fontes da coleta de dados para a análise do presente estudo foram às palestras e mesas redondas, realizadas com os cuidadores dos praticantes do Centro Equoterápico do Campus de Ceres - GO, portadores das patologias de TDAH - Transtorno do Déficit de Atenção e Hiperatividade, TDA - Transtorno de Déficit de Atenção e TEA - Transtorno do Espectro Autista. RESULTADOS E DISCUSSÃO: O profissional de enfermagem pode garantir a continuidade do tratamento em casa e, promover melhores resultados, essa importância do profissional enfermeiro foi percebida durante as palestras e as mesas redondas. CONCLUSÃO: A enfermagem, individualmente, precisa se inserir mais intensamente com o 
desígnio de contribuir no planejamento da assistência e do cuidado do praticante para os familiares e cuidadores podendo abranger uma gama de pessoas que estão envolvidas com as limitações apresentadas.

Palavra-chave: Equoterapia. Cuidador. Enfermagem.

\begin{abstract}
INTRODUCTION: Equine therapy has a wide field of action, aimed at patients with sensory, mental and motor deficiencies, as well as for people who do not adapt to society. OBJECTIVES: To emphasize the importance of nursing attributions in guiding caregivers of equine therapy practitioners at the Equestrian Center of Ceres - GO; evidence the importance of the companion to the patient; guide the caregivers about the characteristics of the pathology, as well as the continued care outside the Equine Therapy Center and elaborate plans for health care and education. METHODOLOGY: The qualitative method of exploratory research was adopted, where we consider the most appropriate for the type of analysis that we intend to carry out. The main sources of data collection for the analysis of the present study were the lectures and round tables held with the caregivers of the Equestrian Center of the Ceres Campus - GO, with the pathologies of ADHD - Disorder of Attention Deficit Hyperactivity Disorder, Attention Deficit Disorder and ASD - Autistic Spectrum Disorder. RESULTS AND DISCUSSION: The nursing professional can guarantee the continuity of the treatment at home and, to promote better results, this importance of the nurse professional was perceived during lectures and round tables. CONCLUSION: Nursing, indiit is necessary to insert more intensely with the intention of contributing in the planning of the care and care of the practitioner to the relatives and caregivers, being able to include a range of people who are involved with the presented limitations.
\end{abstract}

Keyword: Equine therapy. Caregiver.Nursing.

\title{
INTRODUÇÃO
}

Em meados de 2003, quando o Campus Ceres ainda era uma Escola Agrotécnica Federal, deu-se início a discussões sobre a importância da criação de um centro de equoterapia nas dependências da Instituição. Foi então em 2005 que o professor André Luiz de Melo, pertencente ao quadro de funcionários da escola, após ter levado o plano adiante, oficializou-se o Centro de Equoterapia. Desde sua inauguração, o Centro de Equoterapia funciona em companhia com a prefeitura de Ceres-GO, onde a Secretaria Municipal de Saúde de Ceres cede ao Centro de Equoterapia, profissionais habilitados e o IF Goiano colabora com infraestrutura, coordenação, manutenção, equitador, veterinário, treinador e serviços gerais (FEDERAL GOIANO, 2017).

No decorrer do I Seminário Multidisciplinar sobre Equoterapia, em 1999, o qual foi planejado pela Associação Nacional de Equoterapia (ANDE BRASIL, 2011) elaboraram-se 
um conceito de equoterapia, que por sua vez pode ser julgado como uma soma de métodos reeducativos que atuam com o intuito de ultrapassar os danos sensoriais, cognitivos, comportamentais e motores, por meio de uma prática lúdico-desportiva que tem como ferramenta o cavalo (SOUZA; AQUINO; SILVA, 2016).

Seguindo essa linha, a Associação Nacional de Equoterapia (ANDE BRASIL, 2011) também traz seu conceito de Equoterapia, como sendo uma técnica terapêutica e educacional que usa cavalo como um meio de abordagem interdisciplinar, tanto nas áreas de saúde quanto nas áreas de educação e equitação, visando o crescimento biopsicossocial de seus praticantes. O termo Equoterapia foi estabelecido pela mesma com o intuito de identificar as atividades que usam o cavalo como ferramenta para desenvolver práticas com pessoas deficientes ou com necessidades especiais, tendo como foco a reabilitação e a educação das mesmas. (BEZERRA; CARVALHO; BARBOSA, 2011).

A equoterapia tem um vasto campo de atuação, destinando-se à pacientes portadores de deficiências sensoriais, mentais e motoras, além de se destinar às pessoas que não se adaptam á sociedade, por qualquer que sejam as razões, como perturbações emocionais, uso de substâncias psicoativas, entre outras. Sua atuação é desenvolvida tanto na área da saúde quanto nas áreas sociais e educacionais. A equipe idealiza e executa o plano de tratamento de seus praticantes de acordo com as dificuldades e capacidades de cada um, focando assim em suas respectivas áreas de atuação, dentre elas podemos citar a enfermagem (SILVA, 2016).

Em 1995, no Parecer Informativo 004/95, o Conselho Federal de Enfermagem (COFEN) reconheceu na profissão de Enfermagem, que é fundamentada na visão do ser humano como um todo, o constante interesse em utilizar práticas naturais no decorrer do tratamento do cliente, e também as concepções do Código de Ética dos Profissionais de Enfermagem, que possibilita o uso dessas práticas. Entretanto, apenas no ano de 1997, por meio da Resolução 197, que o COFEN reconheceu essas terapias alternativas como qualificação e especialidade do profissional de enfermagem (COFEN, 1997).

Esse reconhecimento da enfermagem possibilitou a integração do enfermeiro dentro da equipe de terapeutas que atuam na equoterapia, onde sua participação pode ter bastante relevância em relação aos cuidadores dos praticantes, assegurando assim a continuação do tratamento em casa. Devido o profissional de enfermagem ter a habilidade de agir junto ao paciente de forma integral, ele pode orientar os cuidadores quanto às necessidades exigentes de cada patologia tratada, visando identificar as formas de cuidado, contribuindo para satisfazer as necessidades humanas básicas (STROCHEIN; RODRIGUES, 2016). 
A atribuição de educar pertencente ao enfermeiro está diretamente relacionada ao desenvolvimento e crescimento de sua profissão. O comprometimento em ensinar foi reconhecido como uma importante responsabilidade do enfermeiro enquanto cuidador, desde que a enfermagem passou a ter reconhecimento como disciplina independente (CHACHKES; CHRIST, 1996).

Nos centros de equoterapia, por serem serviços que promovem assistência aos indivíduos dentro da área da saúde, é de fundamental importância compreender o ponto de vista dos acompanhantes/cuidadores dos praticantes, para assim proporcionar melhores benefícios aos usuários (PRIETSCH, 2012).

$\mathrm{Na}$ maioria das vezes, o cuidador sente-se incapaz e debilitado de agir diante das deficiências de seus entes cuidados, o que influencia diretamente na relação cuidador/paciente. Com base nisso, nota-se indispensável à preocupação em cuidar não somente do praticante, como também desse indivíduo que passa boa parte de seu tempo ofertando seus cuidados (SOUZA; AQUINO; SILVA, 2016).

A palavra cuidador refere-se a um indivíduo de boas qualidades, isto é, um ser com grande amor ao próximo, solidário e com capacidade de doação de si mesmo. Pode ser um membro da família ou de outro meio, que oferta seus cuidados com ou sem gratificação, a pessoas de qualquer que seja a faixa etária, debilitadas tanto fisicamente quanto mentalmente (GUIA PRÁTICO DO CUIDADOR, 2008).

Os praticantes da Equoterapia são caracterizados em várias particularidades, devido a limitações progressivas. Muitas das vezes são deficiências irreversíveis, que requer uma observação prolongada, com necessidade de controle e cuidado. A maneira como o cuidador relaciona-se com o paciente podem minimizar ou proporcionar melhores condições de progressos do mesmo, pelo fato de que ambos são afetados diretamente por esse vínculo criado pelo cuidar. É fundamental que o cuidador compreenda que o tratamento equoterápico também possui grande interferência nessa relação (MEDINA, 2014).

Morano (2003) aponta que o processo de educação do cuidador é o principal ponto de partida para a melhor interação do mesmo com o paciente, fazendo-o entender sua real tarefa no ato do cuidar, ressaltando a necessidade de manter esse cuidado contínuo, e não apenas desenvolvê-lo em específicas situações, ofertando sempre esperança e amor. É indispensável à compreensão de que o acompanhante fornece cuidado não só ao paciente, mas também a ele mesmo, assim como é necessário estar sempre trabalhando novas técnicas de assistência à saúde com o mesmo. 
O presente trabalho teve por objetivo ressaltar a importância das atribuições da enfermagem com os cuidadores dos praticantes do Centro Equoterápico de Ceres-GO, relatando um breve histórico sobre o local da pesquisa, estabelecendo o conceito de equoterapia, apontando assim as principais patologias dos praticantes, para identificar a relação equoterapia $\mathrm{x}$ enfermagem, ressaltando as atribuições do enfermeiro, evidenciando a importância do acompanhante ao paciente, podendo assim orientar os cuidadores sobre as características da patologia, bem como a realização de um cuidado continuado fora do Centro de Equoterapia, para que ocorra a elaboração do plano de cuidados e educação em saúde. Justifica-se esta pesquisa pelo fato do município de Ceres ser hoje considerado um pólo referencial na área da saúde, e pelo fato da equoterapia ser uma nova prática terapêutica, que utiliza o cavalo como ferramenta, através da prática que adota uma técnica que tem sido inserida ao passar dos dias e vêm ocupando cada vez mais o seu espaço, como terapia alternativa, trazendo junto a si o tratamento de vários tipos de distúrbios, com sua eficácia cientificamente comprovada. Podendo assim empregar a enfermagem colaborando no planejamento da assistência e no cuidado tanto com o praticante, como com os cuidadores do Centro Equoterápico de Ceres-GO.

\section{METODOLOGIA}

Trata-se de um estudo com abordagem qualitativa, do tipo exploratório, que busca avaliar os participantes da pesquisa. Entende-se que o viés qualitativo, nesse caso, é o que melhor se enquadra para análise das vivências subjetivas dos cuidadores dos praticantes do Centro Equoterápico. Conforme destaca Chizzotti (1998), essa modalidade metodológica privilegia a consideração dos aspectos individuais e complexos da vida humana, destinando aos significados que os indivíduos dão às suas ações e relações. Gil (1994) salienta que o caráter exploratório de um estudo visa de modo geral o fato investigado.

O estudo foi realizado no Centro de Equoterapia Ceres está integrado ao Instituto Federal Goiano- Campus Ceres, localizado na Rodovia GO-154 - Km 3, S/N - Zona Rural, Ceres - GO, 76300-000, telefone:(62) 3307-7100. Como ponto de partida, o objetivo desta pesquisa foi ressaltar a importância das atribuições da enfermagem com os cuidadores dos praticantes da equoterapia no Centro.Com o apoio da coordenação do Centro foi realizado um levantamento da quantidade de praticantes em ordem decrescente das três patologias específicas que possuem maior número, para que dessa forma fosse atingida uma maior quantidade de cuidadores. As três patologias selecionadas foram elas: TDAH - Transtorno do 
Déficit de Atenção e Hiperatividade, TDA - Transtorno de Déficit de Atenção e TEA Transtorno do Espectro Autista.

A população desta pesquisa incluiu 13 cuidadores dos praticantes do Centro Equoterápico que foram enquadrados nos critérios de inclusão, sendo eles: pacientes que possuem cadastro no Centro de Equoterapia do Campus Ceres; pacientes que necessitem do tratamento; cuidadores mais próximos dos praticantes no Centro de Equoterapia do Campus Ceres; cuidadores que aceitarem a assinar o Termo de Consentimento de Livre Esclarecimento e que concordaram com os procedimentos legais, assinando dessa forma o termo de consentimento. Foi apresentado o TCLE, garantindo aos voluntários que a qualquer momento poderia retirar seu consentimento, sem causar qualquer prejuízo, responsabilidade e penalidade. E como critério de exclusão: pacientes que não possuem cadastro no Centro de Equoterapia do Campus Ceres; pacientes que não necessitem do tratamento e possuem algum déficit; pessoas que não tenham vínculo com as atividades de vida diária dos praticantes do Centro de Equoterapia do Campus Ceres; cuidadores que não aceitarem assinar o TCLE.

As principais fontes da coleta de dados para a análise do presente estudo foram às palestras e as mesas redondas realizadas com os cuidadores dos praticantes do Centro Equoterápico do Campus de Ceres - GO, portadores das patologias de TDA, TDAH e TEA, com o objetivo de ensinar, orientar e implantar cuidados diários voltados para continuidade e melhoria da qualidade de vida dos mesmos. Os cuidadores tiveram a oportunidade de obter maior esclarecimento quanto às patologias específicas, podendo através da ferramenta selecionada, sendo ela: folheto explicativo, contendo orientações de cuidados e atividades a serem executadas fora do Centro, dando assim continuidade ao tratamento e obtendo melhoras no resultado do tratamento. As mesas redondas foram gravadas em áudios, e, posteriormente, transcritas, integralmente, uma a uma. Elas aconteceram em um período de três meses, entre setembro, outubro e novembro de 2017, com duração de 30 a 45 minutos cada uma. Apesar de ter um roteiro de perguntas previamente elaborado, buscamos modificar a direção da conversa quando foi necessário e interessante. As palestras semi-estruturadas, em particular, tornam-se um método eficaz, pois proporciona interesse e passam a ser grandemente utilizadas.

Foi oferecido um período de um mês para que os cuidadores desenvolvessem a implementação dos planos de cuidados contidos nos folhetos explicativos, e dessa forma através da devolutiva nas mesas redondas, analisassem se houve ou não relevância ao projeto executado. Os diálogos diretos realizados nas mesas redondas foram transcritos na íntegra e após tais procedimentos foram feitas leituras criteriosas das falas. Tais leituras visaram à apreensão dos significados contidos nas respostas, onde a partir disso pudesse ser avaliados. 
Dos 13 participantes, foram selecionados 8 que numa visão do conjunto e imersão na narrativa, mais poderiam contribuir com a pesquisa.

Por se tratar de pesquisa envolvendo seres humanos, o projeto foi submetido à aprovação do Comitê de Ética - Centro de Educação Serra da Mesa, sob parecer número 8025.Respeitando-se os preceitos éticos estabelecidos pela resolução 466/12 do Conselho Nacional de Saúde (CNS). Cada participante assinou um termo de consentimento, declarando sua livre participação na pesquisa, após receber os devidos esclarecimentos sobre os objetivos e métodos do presente estudo. Para preservação da identidade dos participantes utilizou-se o código por numeral: Cuidador 1, 2,3,4,5,6,7,8.

Após o tratamento e análise dos resultados surgiram as seguintes categorias que foram assim nominadas: A importância da orientação aos cuidadores sobre as patologias, bem como o cuidado continuado fora do Centro Equoterápico, o Feedback apresentado pelos cuidadores após execução dos planos de cuidados voltados para as atividades diárias, e a relevância da presença diária do profissional de enfermagem atuando como orientador junto aos cuidadores durante a sessão de equoterapia.

\section{RESULTADOS E DISCUSSÃO}

\section{A importância da orientação aos cuidadores}

A equoterapia possibilita que os cuidadores comecem descobrindo e desenvolvendo outras capacidades não identificadas anteriormente no praticante e, desse modo pode-se promover uma nova definição das relações entre eles, o que contribui significativamente ao praticante um melhor ajuste do comportamento. Dessa forma, interrompe ou minimiza o círculo vicioso patológico da relação (SILVA, 2013).Como pode ser comprovado com relato do cuidador um, e dois:

"Tinha muita coisa simples e pequena que eu poderia fazer em casa para melhorar o tratamento, só a falta de informação me fez perder muito tempo. Depois que mudei as rotinas do meu filho, pude perceber uma grande mudança. Eu percebi que quando eu realizo uma atividade segura, eu faço sem stress."CUIDADOR 1.

"A ajuda dos enfermeiros seria interessante nessa questão dos cuidados que a gente deve ter diariamente, com os praticantes, podendo 
assim alcançar maiores possibilidades de evolução.”CUIDADOR 2.

Quando o cuidador se envolve diariamente na participação das atividades diárias com o praticante, os mesmos passam a presenciar o que é possibilitado e o que está em seu alcance a ser realizado para os seus praticantes durante a terapia, os cuidadores conseguem acreditar nas potencialidades dos mesmos de um modo diferente. Experiência estaque passa a se estender ao seio familiar, permitindo assim estimulá-los ainda mais ao modo mais adequado.

Para Lacerda e Oliniski (2004) é importante fortalecer e valorizar o cuidador familiar, mostrando-lhe como é importante seu papel de cuidador e incentivando-o também a cuidar de si. O trabalho profissional com os cuidadores deve contemplar informação e reflexão. Portanto, torna-se notório a importância do preparo dos profissionais que trabalham com a equoterapia para que assim possa lidar com o cuidador, tornando-se necessário que esses profissionais tenham domínio sobre as informações e as percepções dos cuidadores no que diz respeito à ética, à técnica e a sua postura profissional. Dessa forma, permitir á contribuir para que os cuidadores saibam mais sobre a patologia do praticante bem como compreendam às suas reações frente à terapia e as potencialidades que ainda poderão ser exploradas, e assim melhore a qualidade de vida e haja evolução no tratamento do praticante.

\section{Feedback dos cuidadores após execução dos planos de cuidados}

Ao analisar o feedback dos cuidadores após a execução dos planos de cuidados, observou-se que quanto maior a falta de orientação do cuidador frente a seu dependente, maior a necessidade de apoio por parte de um profissional da saúde, sendo o enfermeiro o responsável por essa educação, onde é capaz de identificar um melhor planejamento de práticas educativas que possam dar continuidade ao tratamento fora do Centro.

O desenvolvimento das técnicas nos cuidados de enfermagem possibilita um planejamento de cuidados, onde utilizam vários tipos de aprendizagem como uma linguagem mais adequada. Com ritmo preciso para que cada clientela alcance as informações necessárias, minimizando dessa forma o fracasso em indivíduos e cuidadores que já sofrem limitações. A equipe então planeja tratamentos de forma efetiva para ser efetuada de acordo com as necessidades e potencial de cada praticante (SILVA; 2006), o que pode ser observado com as falas:

"Eu vejo muita vantagem porque eu imagino assim, a educação que ela deu para a gente, é a 
resposta que nós estamos dando hoje para ela..." CUIDADOR. 3

"Tinha dificuldade de agir com a falta de atenção dele e acabava fazendo as tarefas para terminar logo. Agora estou mudando e deixando ele fazer mais sozinho..." CUIDADOR. 4

A esperança de desenvolvimento da qualidade de vida do praticante e do cuidador se constituiu um estímulo, que conduz a evolução dos mesmos, uma vez que por meio da orientação encontraram melhores condições de acesso aos cuidados para aqueles que tem limitações tanto físicas quanto mentais, além da expectativa de melhora do convívio familiar. Deste modo, os cuidadores têm menos sobrecarga no cuidado do praticante, além de maior tempo para o autocuidado, o que pode ser examinado por meio da fala:

“... Eu preciso encontrar as melhores formas de cuidar dela em casa, como ela merece, eu creio que ai então eu terei tempo para viver melhor..."CUIDADOR. 5

O profissional da enfermagem assim como os cuidadores são responsáveis pela organização e prestação de cuidados à pessoa dependente. É preciso um maior envolvimento e responsabilidade das famílias na prestação de cuidados, na maior parte vezes, um nível complexo de conhecimento e habilidade por parte dos familiares cuidadores. Por isso, é necessário que os profissionais de saúde ampliem táticas de ensino adequadas às necessidades concretas dos cuidadores (LANDEIRO et al 2016).

\section{Importância da atuação diária da enfermagem atuando como orientador}

O Conselho Federal de Enfermagem, no Parecer Informativo 004/95 (COFEN, 1995), Reconhece a fundamentação da profissão de Enfermagem, na visão holística do ser humano, o crescente interesse e realizações das práticas naturais no cuidado ao paciente e os aspectos do Código de Ética dos Profissionais de Enfermagem que permitem a utilização das terapias naturais. Finalmente, em 19/03/1997, o COFEN, por meio da Resolução 197 "Estabelece e reconhece as Terapias Alternativas como especialidade e/ou qualificação do profissional de Enfermagem." (COFEN, 1997).

Como sendo parte da equipe de terapeutas multiprofissionais que trabalham na equoterapia, o (a) enfermeiro (a) pode ter um papel de extrema importância no grupo de 
cuidadores e dessa forma elaborar junto à eles um plano de cuidados/terapêutico contribuindo sob a ótica do cuidado e das ações de educação em saúde. Dessa forma, poderá fornecer aos mesmos, informações embasadas sobre as características da patologia que afeta seus filhos nas atividades diárias, para compreensão e cuidados adequados.

Os cuidadores lidam com o déficit de informação e de habilidades, ou seja, onde necessitam de treino de competências. Sendo obrigados, repetidas vezes, a aprender pelo processo da tentativa e erro, levando a falta de confiança e baixa percepção de eficácia, o que impede a mudança para o novo papel. $\mathrm{O}$ cuidador, assim sendo, necessita ser a atenção do enfermeiro podendo assim privilegiar a inserção de intervenções no campo da aprendizagem de habilidades e alcance dos conhecimentos (LANDEIRO et. al 2016).

O profissional de enfermagem pode garantir a continuidade do tratamento fora do Centro e, promover melhores resultados. Essa importância do profissional enfermeiro foi percebida durante as palestras e as mesas redondas, podendo ser identificadas nos fragmentos abaixo:

"Na minha opinião eu acho que é possível sim estar inserido nesta questão que agente ta falando de saúde, então eu acho que dentro, eu não conheço muito as responsabilidades da enfermagem e o que eles podem fazer, mais acredito que é possível sim se colocar,pensar nessa," CUIDADOR 6.

"Achei muito bom as trocas de experiências que tive com as outras mães e também com as enfermeiras, pois tinha coisas que eu não sabia sobre a doença dele"CUIDADOR 7

"Na minha opinião seria muito interessante ter um enfermeiro para participar com a gente em todas as seções de tratamento para ajudar a gente a melhorar os cuidados quando saímos daqui..."CUIDADOR8

$\mathrm{Na}$ equoterapia a equipe necessita ser o mais ampla possível, necessitando ter profissionais habilitados na área da saúde, educação e equitação. Os atendimentos são efetivados através da atenção primária de enfermagem, juntamente com o acompanhamento precedidos pelo diagnostico médico (LANDEIRO et. al 2016).

Os dados deixam claro a necessidade do profissional de enfermagem, nesse contexto e nessa modalidade de tratamento, onde o enfermeiro tem a capacidade de atuar de forma integralizada junto ao praticante, objetivando dessa forma reconhecer as necessidades de 
cuidado e contribuindo para melhor atenderas necessidades humanas básicas. A equoterapia visa à assistência à saúde do indivíduo, seja na prevenção, tratamento ou cura, considerando a pessoa como um todo. Há necessidade de os enfermeiros implementarem programas inovadores de apoio, de informação e formação conduzidas aos familiares cuidadores, para que aconteça melhoria dos serviços de saúde e evolução dos resultados de saúde em nível local.

\section{CONCLUSÃO}

No seguinte trabalho foi possível discutir sobre o cuidador que convive com um praticante que tem limitações físicas e mentais, uma vez que os mesmos estão envolvidos com a melhora no quadro clínico através da implantação do planejamento dos cuidados, podendo entender as limitações do paciente que pratica a equoterapia, orientando os cuidadores sobre as medidas de controle e prevenção das limitações. A relevância da implementação do planejamento de cuidados voltados para os cuidadores dos praticantes de equoterapia é fator positivo, uma vez que a participação e atenção dos mesmos são de suma importância para o desenvolvimento cognitivo, afetivo e social.

A enfermagem, individualmente, precisa se inserir mais intensamente com o desígnio de contribuir no planejamento da assistência e do cuidado do praticante para os familiares e cuidadores podendo abranger uma gama de pessoas que estão envolvidas com as limitações apresentadas.

Ao prestar orientação aos cuidadores, o enfermeiro estará contribuindo tanto para sua capacitação, quanto para uma assistência integral a partir do momento em que a família também é inserida em seu plano de cuidados. E ao cuidar da família, de forma integral, o enfermeiro estará contribuindo para melhoria do quadro clínico do praticante.

Ainda é necessário que sejam realizadas novas pesquisas com amostras maiores e em outras abordagens metodológicas que irão acrescentar e enriquecer a temática que ainda é pouco conhecida, também poderá contribuir para que a presença do enfermeiro nessa prática seja inserida na equipe multiprofissional nas práticas de equoterapia. A partir dos resultados pretende-se elaborar um documento contendo os dados relevantes da pesquisa, dispondo à equipe do Centro de Equoterapia, acreditando que através dos mesmos possa contribuir para a promoção dessas práticas.

Por fim, acredita-se que existe uma lacuna a ser preenchida urgentemente pelo profissional da enfermagem, especialmente pelo enfermeiro, que é um profissional da saúde e sensível por excelência, e que busca constantemente conhecimentos sobre ação e reação do 
indivíduo, a enfermagem, a cada dia vem demonstrando sua importância nos diversos segmentos da saúde, contribuindo com o trabalho da equipe multiprofissional.

Fica o desafio para os cursos de graduação e formação em enfermagem apropriar-se dessas questões no sentido de ocupar esses espaços e dar mais visibilidade a profissão nesse contexto.

\section{REFERÊNCIAS BIBLIOGRÁFICAS}

APOSTILA DO CURSO BÁSICO DE EQUOTERAPIA - ANDE-BRASIL, Agosto 2011.

BEZERRA, MARCUS LOPES; CARVALHO,CINTYA OLIVEIRA; BARBOSA, EVELINE ELOÁ .Equoterapia - tratamento terapêutico na reabilitação de pessoas com necessidades especiais. 32 f.( Dissertação) Especialização do curso de Educação Física da FANOR - Faculdade Nordeste, Fortaleza, CE, 2011.

CHACHKES, ESTHER; CHRIST, GRACE. Questões culturais na educação do paciente. Educação e aconselhamento para pacientes, v. 27, n. 1, p. 13-21, 1996.

CHIZZOTTI, A.Pesquisa em ciências humanas e sociais.São Paulo: Cortez, 1998.

COFEN. Conselho Federal de Enfermagem. Parecer normativo n. 004/95. Dispõe sobre as atividades em Terapia Alternativa. BolInf COREN, v. 18, n. 4, p. 8, 1995.

Resolução 197. Estabelece e reconhece as terapias alternativas como especialidade e/ou qualificação do profissional de Enfermagem. In: Conselho Regional de Enfermagem. Documentos básicos de enfermagem. São Paulo; 1997.

FEDERAL GOIANO, INSTITUTO. Centro de Equoterapia do Campus Ceres completa 11 anos: Solenidade e Dia de Campo compuseram a comemoração do aniversário do Centro, que atende, gratuitamente, 90 pacientes por semana. 2016. Disponível em:<https://www.ifgoiano.edu.br/home/index.php/documentos-servicos-trindade/169destaque-ceres/3508-centro-de-equoterapia-do-campus-ceres-completa-11-anos $>$. Acesso em: 05 jun. 2017.

GIL, A.C.Métodos e técnicas de pesquisa social.São Paulo: atlas, 1994

GUIA PRÁTICO DO CUIDADOR. Série A. Normas e manuais teóricos. Brasília, DF:Ministério da Saúde (Secretaria de Atenção à Saúde/ Secretaria de Gestão do Trabalho e da Educação na Saúde), 2008. 
LACERDA, M. OLINISKI, L. O familiar cuidador e a enfermeira: desenvolvendo interações no contexto domiciliar. Acta Scientiarum. Health Sciences. v. 26, no. 1, p. 239-248. 2004.

LANDEIRO, M. MARTINS, T. PERES, H. Percepção dos enfermeiros sobre Dificuldades e necessidades informacionais dos familiares cuidadores de pessoa dependente. Texto Contexto Enferm. Revista de enfermagem. 2016.

MEDINA, MARIA LECIANA NUNES PINHEIRO. Cuidando de quem cuida: avaliação da Saúde Mental dos cuidadores de crianças em Equoterapia. 2014. 180 p. Dissertação (Mestrado em Psicologia) - Universidade Católica Dom Bosco, Campo Grande, MS, 2014.

MORANO, M. T. A. P. A construção cultural da dependência e impotência: implicações multiprofissional. São Paulo: Loyola, 2003.

PRIETSCH, DOUGLAS RAMOS. Grau de satisfação dos acompanhantes de praticantes de um centro de equoterapia em relação ao serviço oferecido. 2012. 27 p.Dissertação (Especialização em Gestão de Saúde) - Universidade Federal do Rio Grande do Sul, Porto Alegre, RS, 2012.

SILVA, M. C. A percepção das mães de crianças atendidas em Equoterapia. 2006.

SILVA, L. Contribuições de Paulo Freire para a Educação. seduc.mt.gov. 2013.

SOUZA, VANESSA MACEDO DE; AQUINO, GISELlE BRAGA DE; SILVA, ADELIENE OLIVEIRADA. Psicologia e equoterapia: conhecendo as dificuldades enfrentadas pelos cuidadores dos praticantes. Revista Científica da Faminas, v. 7, n. 3, 2016.

STROCHEIN, JÉSSICA REICHERT; RODRIGUES, FRANCISCO CARLOS PINTO. A percepção dos familiares e da equipe sobre o atendimento as crianças com necessidades especiais em um centro de equoterapia.Revista Eletrônica de Extensão da URI.Vol. 12, N.23,2016.

VERGARA, S. C. Projetos e relatórios de pesquisa em administração. São Paulo: Atlas, 2009. 2009. 